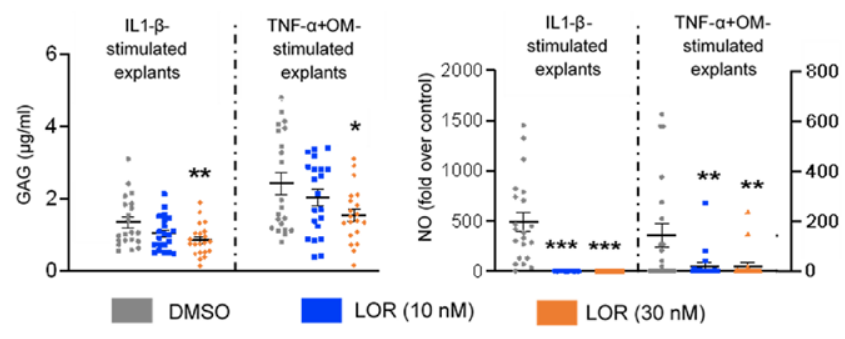

Figure 1. Effects of LOR on the quantity of the cartilage catabolism end products glycosaminoglycan (GAG) and nitric oxide (NO) in supernatants. Knee cartilage explant cultures stimulated with proinflammatory cytokines were subsequently treated with DMSO (control) or LOR as shown. $\mathrm{N}=22$ ${ }^{*} P<0.05,{ }^{* *} P<0.01,{ }^{* * *} P<0.001$ vs. DMSO by one-way ANOVA.

Disclosure of Interests: Vishal Deshmukh Shareholder of: Samumed, LLC, Employee of: Samumed, LLC, Shawn Grogan: None declared, Tim Seo Shareholder of: Samumed, LLC, Employee of: Samumed, LLC, Deepti Bhat Shareholder of: Samumed, LLC, Employee of: Samumed, LLC, William Bugbee: None declared, Darryl D'Lima: None declared, Yusuf Yazici Shareholder of: Samumed, LLC, Grant/research support from: Bristol-Myers Squibb, Celgene, and Genentech, Consultant of: Celgene and Sanofi, Employee of: Samumed, LLC DOI: 10.1136/annrheumdis-2020-eular.6346

\section{AB0071 THERAPEUTIC EFFECTS OF BONE MARROW MESENCHYMAL STEM CELLS-DERIVED EXOSOMES ON OSTEOARTHRITIS}

C. Dong ${ }^{1}$, Y. Liu' ${ }^{1}$, A. Deng ${ }^{1}$, J. Ji ${ }^{1}$, W. Zheng ${ }^{1}$, Z. Gu ${ }^{1} .{ }^{1}$ Affiliated Hospital of Nantong University, Nantong, China

Background: Mesenchymal stem cells (MSCs) have shown chondroprotective effects in clinical models of osteoarthritis $(\mathrm{OA})^{[1]}$.

Objectives: The study aimed to investigate the therapeutic potential of exosomes from human bone marrow MSCs (BM-MSCs) in alleviating OA.

Methods: The anterior cruciate ligament transection (ACLT) anddestabilization of the medial meniscus (DMM) surgery were performed on the knee joints of a rat OA model, followed by intra-articular injection of BM-MSCs or their exosomes. The beneficial effects were evaluated by histological staining, OARSI scores and micro-CT. Furthermore, BM-MSCs-derived exosomes were administrated to primary human chondrocytes to observe the functional and molecular alterations. In addition, IncRNA MEG3 were investigated in chondrocytes to explore the biological contents accounting for anti-OA effects of BM-MSCsderived exosomes.

Results: Based on the observation in the rat OA model, both of BM-MSCs and BM-MSCs-derived exosomes alleviated cartilage destruction, reduced joint damage and restored the trabecular bone of OA rats. In addition, in vitro assays showed that BM-MSCs- exosomes could maintain the chondrocyte phenotype by increasing collagen type II synthesis and inhibiting IL-1 $\beta$ - induced senescence and apoptosis. Furthermore, exosomal IncRNA MEG3 also reduced the senescence and apoptosis of chondrocytes induced by IL- $1 \beta$, indicating that IncRNA MEG3 might partially account for the anti-OA effects of BM-MSC exosomes.

Conclusion: The exosomes from BM-MSCs exerted beneficial therapeutic effects on $\mathrm{OA}$ by reducing the senescence and apoptosis of chondrocytes, suggesting that MSCs-derived exosomes might provide a candidate therapy for OA treatment.

References:

[1] Mckinney J M, Doan T N, Wang L, et al. Therapeutic efficacy of intra-articular delivery of encapsulated human mesenchymal stem cells on early stage osteoarthritis[J]. Eur Cell Mater, 2019, 37: 42-59.

Disclosure of Interests: None declared

DOI: 10.1136/annrheumdis-2020-eular.6040

\section{AB0072 A MULTICOMPONENT MEDICATION PROMOTES CHONDROGENESIS AND REDUCES MMP-13 IN PRIMARY ARTICULAR CHONDROCYTES FROM KNEE OSTEOARTHRITIS PATIENTS IN VITRO}

C. Sanchez ${ }^{1}$, K. Hemmer ${ }^{2}$, N. Kroemmelbein ${ }^{2}$, B. Seilheimer ${ }^{2}$, J. E. Dubuc ${ }^{3}$, C. Antoine ${ }^{4}$, Y. Henrotin ${ }^{1,4} .{ }^{1}$ University of Liège, Bone and Cartilage Research Unit, Liège, Belgium; ${ }^{2}$ Biologische Heilmittel Heel GmbH, Baden-Baden, Germany; ${ }^{3}$ Cliniques Universitaires Saint-Luc, Orthopedic Department, Brussels, Belgium; ${ }^{4}$ Artialis, Liège, Belgium

Background: HE-1100 is a multicomponent medicinal product. Initial preclinical data potentially suggest a preventive effect on cartilage degradation.
Objectives: This study aims to understand the mode of action of HE-1100 on OA chondrocytes in vitro.

Methods: Primary chondrocytes were obtained from 10 knee osteoarthritis (OA) patients undergoing knee replacement surgery. The cultures were treated with $20 \%$ (v/v) HE-1100 or placebo. Samples were collected for subsequent RNA extraction using standard methods. The reads were generated with Illumina NextSeq5000 sequencer and aligned to the human reference genome (UCSC hg19) to generate the transcriptome. Differential expression analysis between $\mathrm{HE}-1100$ and placebo was made in R using the DESeq2 package to identify the differentially expressed genes in the OA-associated regulatory pathways. The protein production of the selected genes was quantified by ELISA in 10 independent human OA chondrocytes cultures.

Results: According to the DESeq2 analysis, HE-1100 significantly modified the expression of 13 genes in OA chondrocytes by at least $10 \%$ with an adjusted p-value < 0.05: EGR1 (+93\%), FOS (+87\%), NR4A1 $(+43 \%)$, DUSP1 $(+18 \%)$, ZFP36 $(+18 \%)$, ZFP36L1 $(+14 \%)$, NFKBIZ $(+16 \%)$ and CYR61 $(+14 \%)$ were upregulated and ATF7IP $(-10 \%)$, TXNIP $(-11 \%)$, C10orf10 (-12\%), CLEC3A $(-12 \%)$ and MMP13 $(-18 \%)$ were downregulated after $24 \mathrm{~h} \mathrm{HE}-1100$ treatment. HE-1100 significantly increased $(2.3$ fold $+/-1.2$ after $24 \mathrm{~h}, \mathrm{p}=0.0444$ and 2.3-fold $+/-1.0$ after $72 \mathrm{~h}, \mathrm{p}=0.0239$ ) the CYR61 protein production by human $\mathrm{OA}$ chondrocytes. After $72 \mathrm{~h}, \mathrm{HE}-1100$ slightly but not significantly increased aggrecan production by $14 \pm 19 \%(p=0.1117)$ and significantly increased type II collagen pro-peptide production by $27 \pm 20 \%(p=0.0147)$. For both time points CYR61 production by OA chondrocytes was positively and significantly correlated with aggrecan $(r=0.66, p=0.0004)$ and type II collagen pro-peptide $(r=0.64, p=0.0008)$ production. In alginate beads culture, pro-MMP- 13 was significantly decreased by HE-1100 treated cultures from day 7 to day 14 (from -16 to $-25 \%, \mathrm{p}<0.05)$ and from day 17 to $21(-22 \%, \mathrm{p}=0.0331)$ in comparison to controls.

Conclusion: HE-1100 significantly modified the expression of DUSP1, C10orf10, ZFP36/L1 and CLEC3A, which are pathway mediators involved in MMP-13 expression and activation. Further, long-term (28 days) treatment with $\mathrm{HE}-1100$ significantly reduced the production of pro-MMP-13, the inactive precursor of the metalloproteinase MMP-13 involved in type II collagen degradation. HE-1100 also promoted extracellular matrix formation probably through CYR61 production, a growth factor well correlated with type II collagen and aggrecan production.

References: /

Acknowledgments: We would like to thank the staff of the GIGA ULiège Genomic Next Generation Sequencing platform for performing the RNA sequencing and Benoit Charloteaux for his help in RNAseq data analysis.

Disclosure of Interests: christelle sanchez: None declared, Kathrin Hemmer Employee of: Heel, Natascha Kroemmelbein Employee of: Heel, Bernd Seilheimer Employee of: Heel, Jean-Emile Dubuc: None declared, Christophe Antoine Employee of: Artialis, Yves Henrotin Grant/research support from: HEEL, TILMAN

DOI: 10.1136/annrheumdis-2020-eular.2106

\section{AB0073 BOSWELLIA SERRATA EXTRACT AND CURCUMIN INCREASE GDF15 PRODUCTION BY HUMAN PRIMARY OSTEOARTHRITIS CHONDROCYTES: A NEW MECHANISM OF ACTION}

C. Sanchez ${ }^{1}$, J. Zappia ${ }^{1}$, Y. Dierckxsens ${ }^{2}$, J. P. Delcour ${ }^{3}$, Y. Henrotin ${ }^{1,4}$. ${ }^{1}$ University of Liège, Bone and Cartilage Research Unit, Liège, Belgium;

${ }^{2}$ Tilman, Baillonville, Belgium; ${ }^{3}$ Centre Hospitalier du Bois de l'Abbaye, Seraing, Belgium; ${ }^{4}$ Princess Paola Hospital, Marche-en-Famenne, Belgium

Background: Boswellia serrata extract (BSE) and curcumin are used to relief symptoms in osteoarthritis (OA).

Objectives: This study aims to better understand the mode of action of these compounds on OA chondrocytes in vitro.

Methods: Therapeutic plasmatic concentrations of the different components of BSE correspond to an in vitro range from 25 to $100 \mu \mathrm{g} / \mathrm{ml}$ of total BSE $(100 \mu \mathrm{g} / \mathrm{ml}$ of BSE corresponds to $9.2 \mu \mathrm{M}$ of $11-\mathrm{keto}-\beta$-boswellic acid (KBA), $5.2 \mu \mathrm{M}$ of acetylKBA, $22 \mu \mathrm{M}$ de aBA, $34 \mu \mathrm{M}$ de $\beta B A, 4.4 \mu \mathrm{M}$ de acetylaBA and 13.2 acetyl $\beta B A$ ), and between 2 to $10 \mu \mathrm{M}$ for bioavaibility-increased curcumin. BSE $(5-100 \mu \mathrm{g} / \mathrm{ml})$ and curcumin $(0.04$ to $4 \mu \mathrm{g} / \mathrm{ml}$ corresponding to 0.1 to $10 \mu \mathrm{M}$ ) were tested separately on primary chondrocytes from 3 OA patients. Lactate Deshydrogenase $\mathrm{LDH}$, nitrite $\left(\mathrm{NO}_{2}\right)$ interleukin (IL)-6 and Growth Differentiation Factor (GDF)15 were quantified in $72 \mathrm{~h}$-treated supernatant using enzyme activity, Griess reaction and ELISAs, respectively.

Results: No mortality was observed at the tested concentrations. BSE and curcumin both decreased concentration-dependently $\mathrm{NO}_{2}$ and IL-6 production, and increased GDF15 production. For $\mathrm{NO}_{2}$ production, the decrease was observed from $0.2 \mu \mathrm{g} / \mathrm{ml}$ of curcumin and $10 \mu \mathrm{g} / \mathrm{ml}$ of BSE. For IL-6 production, 